\title{
Presic Type Fixed Point Theorem for Four Maps in Metric Spaces
}

\author{
K. P. R. Rao, ${ }^{1}$ Sk. Sadik, ${ }^{2}$ and S. Manro ${ }^{3}$ \\ ${ }^{1}$ Department of Mathematics, Acharya Nagarjuna University, Nagarjuna Nagar 522 510, India \\ ${ }^{2}$ Department of Mathematics, Sir C.R.R. College of Engineering, Eluru 534 007, India \\ ${ }^{3}$ Department of Mathematics, Thapar University, Patiala, India
}

Correspondence should be addressed to S. Manro; sauravmanro@hotmail.com

Received 2 July 2016; Accepted 27 October 2016

Academic Editor: Kaleem R. Kazmi

Copyright (C) 2016 K. P. R. Rao et al. This is an open access article distributed under the Creative Commons Attribution License, which permits unrestricted use, distribution, and reproduction in any medium, provided the original work is properly cited.

We obtained a Presic type fixed point theorem for two pairs of jointly $2 k$-weakly compatible maps in metric spaces. We also have given an example to illustrate our main theorem.

\section{Introduction and Preliminaries}

Banach [1], in 1932, proved the following theorem, popularly known as Banach contraction principle.

Theorem 1. Let $(X, d)$ be a complete metric space and let $f$ be a self-mapping on $X$ satisfying $d(f x, f y) \leq k d(x, y)$ for all $x, y \in X$, where $0 \leq k<1$. Then $f$ has a unique fixed point in $X$.

There are number of generalizations of such principle (see [2-12]). One such generalization is given by Presic [13] in 1965.

Let $f: X^{k} \rightarrow X$, where $k \geq 1$ is a positive integer. A point $x^{*} \in X$ is called a fixed point of $f$ if $x^{*}=f\left(x^{*}, x^{*}, \ldots, x^{*}\right)$. Consider the $k$-order nonlinear difference equation

$$
\begin{aligned}
& x_{n+1}=f\left(x_{n-k+1}, x_{n-k+2}, \ldots, x_{n}\right) \\
& \qquad \text { for } n=k-1, k, k+1, \ldots .
\end{aligned}
$$

with the initial values $x_{0}, x_{1}, x_{2}, \ldots, x_{k-1} \in X$.

Equation (1) can be studied by means of fixed point theory in view of the fact that $x \in X$ is a solution of (1) if and only if $x$ is a fixed point of $f$. One of the most important results in this direction is obtained by Presic [13] in the following way.
Theorem 2 (see [13]). Let $(X, d)$ be a complete metric space, $k$ be a positive integer, and $f: X^{k} \rightarrow X$. Suppose that

$$
\begin{aligned}
& d\left(f\left(x_{1}, x_{2}, \ldots, x_{k}\right), f\left(x_{2}, x_{3}, \ldots, x_{k+1}\right)\right) \\
& \quad \leq \sum_{i=1}^{k} q_{i} d\left(x_{i}, x_{i+1}\right)
\end{aligned}
$$

holds for all $x_{1}, x_{2}, \ldots, x_{k}, x_{k+1} \in X$, where $q_{i} \geq 0$ and $\sum_{i=1}^{k} q_{i} \in[0,1)$. Then $f$ has a unique fixed point $x^{*}$. Moreover, for any arbitrary points $x_{1}, x_{2}, \ldots, x_{k+1}$ in $X$, the sequence $\left\{x_{n}\right\}$ defined by $x_{n+k}=f\left(x_{n}, x_{n+1}, \ldots, x_{n+k-1}\right)$, for all $n \in N$ converges to $x^{*}$.

Later Ciric and Presic [14] generalized the above theorem as follows.

Theorem 3 (see [14]). Let $(X, d)$ be a complete metric space, $k$ be a positive integer, and $f: X^{k} \rightarrow X$. Suppose that

$$
\begin{aligned}
& d\left(f\left(x_{1}, x_{2}, \ldots, x_{k}\right), f\left(x_{2}, x_{3}, \ldots, x_{k+1}\right)\right) \\
& \quad \leq \lambda \max \left\{d\left(x_{i}, x_{i+1}\right): 1 \leq i \leq k\right\}
\end{aligned}
$$

holds for all $x_{1}, x_{2}, \ldots, x_{k}, x_{k+1}$ in $X$, where $\lambda \in[0,1)$. Then $f$ has a fixed point $x^{*} \in X$. Moreover, for any arbitrary points $x_{1}, x_{2}, \ldots, x_{k+1}$ in $X$, the sequence $\left\{x_{n}\right\}$ defined by $x_{n+k}=f\left(x_{n}, x_{n+1}, \ldots, x_{n+k-1}\right)$, for all $n \in N$ converges to $x^{*}$. 
Moreover, if $d(f(u, u, \ldots, u), f(v, v, \ldots, v))<d(u, v)$ holds for all $u, v \in X$ with $u \neq v$, then $x^{*}$ is the unique fixed point of $f$.

Recently Rao et al. [2, 3] obtained some Presic type theorems for two and three maps in metric spaces. Now we give the following definition of $[2,3]$.

Definition 4. Let $X$ be a nonempty set and $T: X^{2 k} \rightarrow X$ and $f: X \rightarrow X$. The pair $(f, T)$ is said to be $2 k$-weakly compatible if $f(T(x, x, \ldots, x, x))=T(f x, f x, \ldots, f x, f x)$ whenever $x \in$ $X$ such that $f x=T(x, x, \ldots, x, x)$.

Using this definition, Rao et al. [2] proved the following.

Theorem 5 (see [2]). Let $(X, d)$ be a metric space, $k$ be a positive integer, and $S, T: X^{2 k} \rightarrow X, f: X \rightarrow X$ be mappings satisfying

$(1.4 .1) d\left(S\left(x_{1}, x_{2}, \ldots, x_{2 k}\right), T\left(x_{2}, x_{3}, \ldots, x_{2 k+1}\right)\right) \leq \lambda \max \left\{d\left(f x_{i}\right.\right.$, $\left.f\left(x_{i+1}\right): 1 \leq i \leq 2 k\right\}$ for all $x_{1}, x_{2}, \ldots, x_{2 k}, x_{2 k+1}$ in $X$,

(1.4.2) $d\left(T\left(y_{1}, y_{2}, \ldots, y_{2 k}\right), S\left(y_{2}, y_{3}, \ldots, y_{2 k+1}\right)\right) \leq \lambda \max \left\{d\left(f y_{i}\right.\right.$, $\left.\left.f y_{i+1}\right): 1 \leq i \leq 2 k\right\}$ for all $y_{1}, y_{2}, \ldots, y_{2 k}, y_{2 k+1}$ in $X$, where $0 \leq \lambda<1$,

(1.4.3) $d(S(u, \ldots, u), T(v, \ldots, v))<d(f u, f v)$, for all $u, v \in X$ with $u \neq v$.

(1.4.4) Suppose that $f(X)$ is complete and either $(f, S)$ or $(f, T)$ is a $2 k$-weakly compatible pair.

Then there exists a unique point $p \in X$ such that $f p=p=$ $S(p, \ldots, p)=T(p, \ldots, p)$. pairs.

Now we give a definition of jointly $2 k$-weakly compatible

Definition 6. Let $X$ be a nonempty set, $k$ be a positive integer, $S, T: X^{2 k} \rightarrow X$, and $f, g: X \rightarrow X$. The pairs $(f, S)$ and $(g, T)$ are said to be jointly $2 k$-weakly compatible pairs if $f(S(x, x, \ldots, x))=S(f x, f x, \ldots, f x))$ and $g(T(x, x, \ldots, x))=$ $T(g x, g x, \ldots, g x))$ whenever there exists $x \in X$ such that $f x=S(x, x, \ldots, x)$ and $g x=T(x, x, \ldots, x)$.

In this paper, we obtain a Presic type theorem for four mappings satisfying a slight different contractive condition in metric spaces. We also give an example and two corollaries to our main theorem.

Now we prove our main result.

\section{Main Result}

Theorem 7. Let $(X, d)$ be a complete metric space, $k$ be a positive integer, and $S, T: X^{2 k} \rightarrow X$ and $f, g: X \rightarrow X$ be mappings satisfying

(2.1.1) $S\left(X^{2 k}\right) \subseteq g(X), T\left(X^{2 k}\right) \subseteq f(X)$,

(2.1.2) $d\left(S\left(x_{1}, x_{2}, \ldots, x_{2 k-1}, x_{2 k}\right), T\left(y_{1}, y_{2}, \ldots, y_{2 k-1}, y_{2 k}\right)\right) \leq$ $\lambda \max \left\{d\left(g x_{1}, f y_{1}\right), d\left(f x_{2}, g y_{2}\right), d\left(g x_{3}, f y_{3}\right), d\left(f x_{4}\right.\right.$, $\left.\left.g y_{4}\right), \ldots, d\left(g x_{2 k-1}, f y_{2 k-1}\right), d\left(f x_{2 k}, g y_{2 k}\right)\right\}$ for all $x_{1}, x_{2}, \ldots, x_{2 k}, y_{1}, y_{2}, \ldots, y_{2 k} \in X$ and $0<\lambda<$ 1 ,
(2.1.3) $(f, S)$ and $(g, T)$ are jointly $2 k$-weakly compatible pairs.

(2.1.4) Suppose $z=f p=g p$ for some $p \in X$ whenever there exists a sequence $\left\{y_{2 k+n}\right\}_{n=1}^{\infty}$ in $X$ such that $\lim _{n \rightarrow \infty} y_{2 k+n}=z \in X$

Then $z$ is the unique point in $X$ such that $z=f z=$ $g z=s(z, z, \ldots, z, z)=T(z, z, \ldots, z, z)$.

Proof. Suppose $x_{1}, x_{2}, \ldots, x_{2 k}$ are arbitrary points in $X$. From (2.1.1), define

$$
\begin{aligned}
y_{2 k+2 n-1}=S\left(x_{2 n-1}, x_{2 n}, \ldots, x_{2 k+2 n-2}\right) & =g x_{2 k+2 n-1} \\
y_{2 k+2 n}=T\left(x_{2 n}, x_{2 n+1}, \ldots, x_{2 k+2 n-1}\right) & =f x_{2 k+2 n} \\
& \text { for } n=1,2, \ldots .
\end{aligned}
$$

Let

$$
\begin{aligned}
\alpha_{2 n} & =d\left(f x_{2 n}, g x_{2 n+1}\right), \\
\alpha_{2 n-1} & =d\left(g x_{2 n-1}, f x_{2 n}\right), \\
& n=1,2, \ldots .
\end{aligned}
$$

Let $\theta=\lambda^{1 / 2 k}$ and $\mu=\max \left\{\alpha_{1} / \theta, \alpha_{2} / \theta^{2}, \ldots, \alpha_{2 k} / \theta^{2 k}\right\}$.

Then $0<\theta<1$ and by selection of $\mu$ we have

$$
\begin{aligned}
& \alpha_{n} \leq \mu \theta^{n}, \quad \text { for } n=1,2, \ldots, 2 k . \\
& \alpha_{2 k+1}=d\left(g x_{2 k+1}, f x_{2 k+2}\right)=d\left(S \left(x_{1}, x_{2}, x_{3}, x_{4}, \ldots,\right.\right. \\
& \left.\left.x_{2 k-1}, x_{2 k}\right), T\left(x_{2}, x_{3}, x_{4}, \ldots, x_{2 k}, x_{2 k+1}\right)\right) \leq \lambda \\
& \cdot \max \left\{d\left(g x_{1}, f x_{2}\right), d\left(f x_{2}, g x_{3}\right), d\left(g x_{3}, f x_{4}\right),\right. \\
& \left.d\left(f x_{4}, g x_{5}\right), \ldots, d\left(g x_{2 k-1}, f x_{2 k}\right), d\left(f x_{2 k}, g x_{2 k+1}\right)\right\} \\
& \quad \text { from }(2.1 .2)
\end{aligned}
$$

$=\lambda \max \left\{\alpha_{1}, \alpha_{2}, \alpha_{3}, \alpha_{4}, \ldots, \alpha_{2 k-1}, \alpha_{2 k}\right\} \leq \lambda \max \{\mu \theta$,

$\left.\mu \theta^{2}, \mu \theta^{3}, \mu \theta^{4}, \ldots, \mu \theta^{2 k-1}, \mu \theta^{2 k}\right\} \quad$ from (6)

$=\lambda \mu \theta=\theta^{2 k} \mu \theta=\mu \theta^{2 k+1}$,

$\alpha_{2 k+2}=d\left(f x_{2 k+2}, g x_{2 k+3}\right)=d\left(S\left(x_{3}, x_{4}, x_{5}, x_{6}, \ldots\right.\right.$,

$\left.\left.x_{2 k+1}, x_{2 k+2}\right), T\left(x_{2}, x_{3}, x_{4}, \ldots, x_{2 k}, x_{2 k+1}\right)\right) \leq \lambda$

- $\max \left\{d\left(g x_{3}, f x_{2}\right), d\left(f x_{4}, g x_{3}\right), d\left(g x_{5}, f x_{4}\right)\right.$,

$d\left(f x_{6}, g x_{5}\right), \ldots, d\left(g x_{2 k+1}, f x_{2 k}\right)$,

$\left.d\left(f x_{2 k+2}, g x_{2 k+1}\right)\right\} \quad$ from $(2.1 .2)$

$=\lambda \max \left\{\alpha_{2}, \alpha_{3}, \alpha_{4}, \alpha_{5}, \ldots, \alpha_{2 k}, \alpha_{2 k+1}\right\} \leq \lambda \max \left\{\mu \theta^{2}\right.$,

$\left.\mu \theta^{3}, \mu \theta^{4}, \mu \theta^{5}, \ldots, \mu \theta^{2 k}, \mu \theta^{2 k+1}\right\} \quad$ from (6), (7)

$=\lambda \mu \theta^{2}=\theta^{2 k} \mu \theta^{2}=\mu \theta^{2 k+2}$. 
Continuing in this way, we get

$$
\alpha_{n} \leq \mu \theta^{n} \text { for } n=1,2, \ldots
$$

Now consider

$$
\begin{aligned}
& d\left(y_{2 k+2 n-1}, y_{2 k+2 n}\right)=d\left(S \left(x_{2 n-1}, x_{2 n}, x_{2 n+1}, x_{2 n+2}, \ldots,\right.\right. \\
& \left.x_{2 k+2 n-3}, x_{2 k+2 n-2}\right), T\left(x_{2 n}, x_{2 n+1}, x_{2 n+2}, x_{2 n+3}, \ldots,\right. \\
& \left.\left.x_{2 k+2 n-2}, x_{2 k+2 n-1}\right)\right) \leq \lambda \max \left\{d\left(g x_{2 n-1}, f x_{2 n}\right),\right. \\
& d\left(f x_{2 n}, g x_{2 n+1}\right), d\left(g x_{2 n+1}, f x_{2 n+2}\right), \\
& d\left(f x_{2 n+2}, g x_{2 n+3}\right), \ldots, d\left(g x_{2 k+2 n-3}, f x_{2 k+2 n-2}\right), \\
& \left.d\left(f x_{2 k+2 n-2}, g x_{2 k+2 n-1}\right)\right\}=\lambda \max \left\{\alpha_{2 n-1}, \alpha_{2 n}, \alpha_{2 n+1},\right. \\
& \left.\alpha 2 n+2, \ldots, \alpha_{2 k+2 n-3}, \alpha_{2 k+2 n-2}\right\} \leq \lambda \max \left\{\mu \theta^{2 n-1}, \mu \theta^{2 n},\right. \\
& \left.\mu \theta^{2 n+1}, \mu \theta^{2 n+2}, \ldots, \mu \theta^{2 k+2 n-3}, \mu \theta^{2 k+2 n-2}\right\} \quad \text { from }(9) \\
& =\lambda \mu \theta^{2 n-1}=\theta^{2 k} \mu \theta^{2 n-1}=\mu \theta^{2 k+2 n-1} .
\end{aligned}
$$

Also

$$
\begin{aligned}
& d\left(y_{2 k+2 n}, y_{2 k+2 n+1}\right)=d\left(S \left(x_{2 n+1}, x_{2 n+2}, x_{2 n+3}, x_{2 n+4}, \ldots,\right.\right. \\
& \left.x_{2 k+2 n-1}, x_{2 k+2 n}\right), T\left(x_{2 n}, x_{2 n+1}, x_{2 n+2}, x_{2 n+3}, \ldots,\right. \\
& \left.\left.x_{2 k+2 n-2}, x_{2 k+2 n-1}\right)\right) \leq \lambda \max \left\{d\left(g x_{2 n+1}, f x_{2 n}\right),\right. \\
& d\left(f x_{2 n+2}, g x_{2 n+1}\right), d\left(g x_{2 n+3}, f x_{2 n+2}\right), \\
& d\left(f x_{2 n+4}, g x_{2 n+3}\right), \ldots, d\left(g x_{2 k+2 n-1}, f x_{2 k+2 n-2}\right), \\
& \left.d\left(f x_{2 k+2 n}, g x_{2 k+2 n-1}\right)\right\}=\lambda \max \left\{\alpha_{2 n}, \alpha_{2 n+1}, \alpha_{2 n+2},\right. \\
& \left.\alpha_{2 n+3}, \ldots, \alpha_{2 k+2 n-2}, \alpha_{2 k+2 n-1}\right\} \leq \lambda \max \left\{\mu \theta^{2 n}, \mu \theta^{2 n+1},\right. \\
& \left.\mu \theta^{2 n+2}, \mu \theta^{2 n+3}, \ldots, \mu \theta^{2 k+2 n-2}, \mu \theta^{2 k+2 n-1}\right\} \quad \text { from }(9) \\
& =\lambda \mu \theta^{2 n}=\theta^{2 k} \mu \theta^{2 n}=\mu \theta^{2 k+2 n} .
\end{aligned}
$$

Thus from (10) and (11) we have

$$
d\left(y_{2 k+n}, y_{2 k+n+1}\right) \leq \mu \theta^{2 k+n} \text { for } n=1,2,3, \ldots
$$

Now from $m>n$ consider

$$
\begin{aligned}
d\left(y_{2 k+n}, y_{2 k+m}\right) \leq & d\left(y_{2 k+n}, y_{2 k+n+1}\right) \\
& +d\left(y_{2 k+n+1}, y_{2 k+n+2}\right) \\
& +d\left(y_{2 k+n+2}, y_{2 k+n+3}\right)+\cdots \\
& +d\left(y_{2 k+m-1}, y_{2 k+m}\right) \\
\leq & \mu \theta^{2 k+n}+\mu \theta^{2 k+n+1}+\mu \theta^{2 k+n+2}+\cdots \\
& +\mu \theta^{2 k+m-1} \text { from }(12) \\
\leq & \mu \theta^{2 k} \frac{\theta^{n}}{1-\theta} \longrightarrow 0
\end{aligned}
$$

Hence $\left\{y_{2 k+n}\right\}$ is a Cauchy sequence in $X$. Since $X$ is complete there exists $z \in X$ such that $y_{2 k+n} \rightarrow z$ as $n \rightarrow \infty$. From (2.1.4), there exists $u \in X$ such that

$$
z=f u=g u
$$

Now consider

$$
\begin{aligned}
& d\left(S(u, u, \ldots, u, u), y_{2 k+2 n}\right)=d(S(u, u, \ldots, u, u), \\
& \left.\quad T\left(x_{2 n}, x_{2 n+1}, \ldots, x_{2 k+2 n-2}, x_{2 k+2 n-3}\right)\right) \leq \lambda \\
& \cdot \max \left\{d\left(g p, f x_{2 n}\right), d\left(f p, g x_{2 n+1}\right), d\left(g p, f x_{2 n+2}\right),\right. \\
& \quad d\left(f p, g x_{2 n+3}\right), \ldots, d\left(g p, f x_{2 k+2 n-2}\right), \\
& \left.d\left(f p, g x_{2 k+2 n-1}\right)\right\} .
\end{aligned}
$$

Letting $n \rightarrow \infty$ and using $(14) d(S(u, u, \ldots, u, u), f u) \leq 0$ so that

$$
f u=S(u, u, \ldots, u, u)
$$

Similarly we have

$$
f u=g u=T(u, u, \ldots, u, u) .
$$

Since $(f, S)$ and $(g, T)$ are jointly $2 k$-weakly compatible pairs, we have

$$
\begin{aligned}
& f z=f(f u)=f(S(u, u, \ldots, u, u))=S(f u, f u, \ldots, \\
& \quad f u, f u)=S(z, z, \ldots, z, z), \\
& g z=T(z, z, \ldots, z, z) \\
& d(f z, z)=d(f z, g u)=d(S(z, z, \ldots, z, z), T(u, u, \\
& \quad \ldots, u, u)) \quad \text { from }(18),(17) \\
& \leq \lambda \max \{d(g z, f u), d(f z, g u), d(g z, f u), d(f z, g u), \\
& \quad \ldots, d(g z, f u), d(f z, g u)\}=\lambda \max \{d(g z, z), \\
& d(f z, z)\} \quad \text { from }(14) .
\end{aligned}
$$

Similarly, we have

$$
d(g z, z) \leq \lambda \max \{d(z, f z), d(z, g z)\} .
$$

From (20) and (21) we have

$$
\begin{aligned}
\max & \{d(z, f z), d(z, g z)\} \\
\leq \lambda \max & \{d(z, f z), d(z, g z)\}
\end{aligned}
$$

which in turn yields that

$$
\begin{aligned}
& f z=z, \\
& g z=z .
\end{aligned}
$$

From (18), (19), and (23), we have

$$
z=f z=g z=S(z, z, \ldots, z, z)=T(z, z, \ldots, z, z) .
$$


Suppose there exists $z^{\prime} \in X$ such that

$$
\begin{aligned}
z^{\prime} & =f z^{\prime}=g z^{\prime}=S\left(z^{\prime}, z^{\prime}, \ldots, z^{\prime}, z^{\prime}\right) \\
& =T\left(z^{\prime}, z^{\prime}, \ldots, z^{\prime}, z^{\prime}\right) .
\end{aligned}
$$

Then from (2.1.2) we have

$$
\begin{aligned}
& d\left(z, z^{\prime}\right)=d\left(S(z, z, \ldots, z, z), T\left(z^{\prime}, z^{\prime}, \ldots, z^{\prime}, z^{\prime}\right)\right) \\
& \quad \leq \lambda \max \left\{d\left(g z, f z^{\prime}\right), d\left(f z, g z^{\prime}\right), \ldots, d\left(g z, f z^{\prime}\right)\right. \\
& \left.d\left(f z, g z^{\prime}\right)\right\}=\lambda d\left(z, z^{\prime}\right) .
\end{aligned}
$$

This implies that $z^{\prime}=z$.

Thus $z$ is the unique point in $X$ satisfying (24).

Now we give an example to illustrate our main Theorem 7.

Example 8. Let $X=[0,1], k=1$, and $d(x, y)=|x-y|$. Define $S(x, y)=\left(3 x^{2}+2 y\right) / 72, T(x, y)=\left(2 x+3 y^{2}\right) / 72, f x=x / 6$, and $g x=x^{2} / 4$ for all $x, y \in X$.

Then, for all $x_{1}, x_{2}, y_{1}, y_{2} \in X$, we have

$$
\begin{aligned}
& d\left(S\left(x_{1}, x_{2}\right), T\left(y_{1}, y_{2}\right)\right)=\left|\frac{3 x_{1}^{2}+2 x_{2}}{72}-\frac{2 y_{1}+3 y_{2}^{2}}{72}\right| \\
& \quad=\frac{1}{72}\left|\left(3 x_{1}^{2}-2 y_{1}\right)+\left(2 x_{2}-3 y_{2}^{2}\right)\right| \\
& \quad \leq \frac{1}{72}\left(\left|3 x_{1}^{2}-2 y_{1}\right|+\left|2 x_{2}-3 y_{2}^{2}\right|\right) \\
& \quad \leq \frac{1}{36} \max \left\{\left|3 x_{1}^{2}-2 y_{1}\right|,\left|2 x_{2}-3 y_{2}^{2}\right|\right\} \\
& \quad=\frac{1}{3} \max \left\{d\left(g x_{1}, f y_{1}\right), d\left(f x_{2}, g y_{2}\right)\right\} .
\end{aligned}
$$

Thus the condition (2.1.2) of Theorem 7 is satisfied. One can easily verify the remaining conditions of Theorem 7. Clearly, 0 is the unique point in $X$ such that $0=f 0=g 0=$ $S(0,0, \ldots, 0,0)=T(0,0, \ldots, 0,0)$.

Corollary 9. Let $(X, d)$ be a metric space and $S, T: X^{2 k} \rightarrow X$ and $f: X \rightarrow X$ be mappings satisfying

(2.3.1) $S\left(X^{2 k}\right) \subseteq f(X), T\left(X^{2 k}\right) \subseteq f(X)$,

(2.3.2) $d\left(S\left(x_{1}, x_{2}, \ldots, x_{2 k-1}, x_{2 k}\right), T\left(y_{1}, y_{2}, \ldots, y_{2 k-1}, y_{2 k}\right)\right) \leq$ $\lambda \max \left\{d\left(f x_{i}, f y_{i}\right) / 1 \leq i \leq 2 k\right\}$ for all $x_{1}, x_{2}, \ldots, x_{2 k}$, $y_{1}, y_{2}, \ldots, y_{2 k} \in X$ and $0<\lambda<1$,

(2.3.3) $f(X)$ is a complete subspace of $X$,

(2.3.4) the pairs $(f, S)$ or $(f, T)$ is $2 k$-weakly compatible.

Then there exists a unique $u \in X$ such that $u=f u=S(u, u$, $\ldots, u, u)=T(u, u, \ldots, u, u)$.

Corollary 10. Let $(X, d)$ be a complete metric space and $S, T$ : $X^{2 k} \rightarrow X$ be mappings satisfying

(2.4.1) $d\left(S\left(x_{1}, x_{2}, \ldots, x_{2 k-1}, x_{2 k}\right), T\left(y_{1}, y_{2}, \ldots, y_{2 k-1}, y_{2 k}\right)\right) \leq$ $\lambda \max \left\{d\left(x_{i}, y_{i}\right) / 1 \leq i \leq 2 k\right\}$ for all $x_{1}, x_{2}, \ldots, x_{2 k}, y_{1}$, $y_{2}, \ldots, y_{2 k} \in X$ and $0<\lambda<1$.
Then there exists a unique $u \in X$ such that $u=S(u, u, \ldots, u)=$ $T(u, u, \ldots, u)$.

Remark 11. Corollaries 9 and 10 are slight variant of theorems of $[2,3]$.

\section{Competing Interests}

The authors declare that there are no competing interests regarding the publication of this paper.

\section{References}

[1] S. Banach, Theorie des Operations Lineaires, Manograic Mathematic Zne, Warsaw, Poland, 1932.

[2] K. P. R. Rao, G. N. V. Kishore, and M. Mushtaq Ali, "A generalization of the Banach contraction principle of Presic type for three maps," Mathematical Sciences, vol. 3, no. 3, pp. 273280, 2009.

[3] K. P. Rao, M. Mustaq Ali, and B. Fisher, "Some Presic type generalizations of the Banach contraction principle," Mathematica Moravica, vol. 15, no. 1, pp. 41-47, 2011.

[4] R. P. Agarwal, M. Meehan, and D. O'Regan, Fixed Point Theory and Applications, vol. 141, Cambridge University Press, Cambridge, UK, 2001.

[5] R. P. Agarwal, D. O'Regan, and D. R. Sahu, Fixed Point Theory for Lipschitzian-type Mappings with Applications, vol. 6 of Topological Fixed Point Theory and Its Applications, Springer, New York, NY, USA, 2009.

[6] R. Kannan, "Some results on fixed points," Bulletin of the Calcutta Mathematical Society, vol. 60, pp. 71-76, 1968.

[7] J. Schauder, "Fixed point theorem in continuous mapping," Mathematische Annalen, vol. 174, pp. 285-290, 1930.

[8] S. K. Chatterjea, "Fixed point theorems," Comptes Rendus de l'Academie Bulgare des Sciences, vol. 25, pp. 727-730, 1972.

[9] B. E. Rhoades, "A comparison of various definitions of contractive mappings," Transactions of the American Mathematical Society, vol. 226, pp. 257-290, 1977.

[10] B. E. Rhoades, Contractive Definitions-Nonlinear Analysis, World Scientific Publishing Company, New Jersey, NJ, USA, 1998.

[11] T. Zamfirescu, "Fix point theorems in metric spaces," Archiv der Mathematik, vol. 23, pp. 292-298, 1972.

[12] I. A. Rus, "Generalized Contractions, Seminar on fixed point theory," University of Cluj-Napoca, vol. 3, pp. 1-130, 1983.

[13] S. B. Presic, "Sur une classe $d^{1}$ inequations aux differences finite et sur la convergence de certaines suites," Publications de l'Institut Mathématique, vol. 5, no. 19, pp. 75-78, 1965.

[14] L. B. Ciric and S. B. Presic, "On Presic type generalization of Banach contraction mapping principle," Acta Mathematica Universitatis Comenianae, vol. 76, no. 2, pp. 143-147, 2007. 


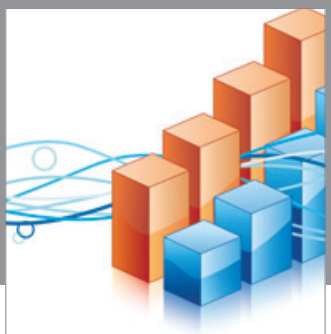

Advances in

Operations Research

vatem alat4

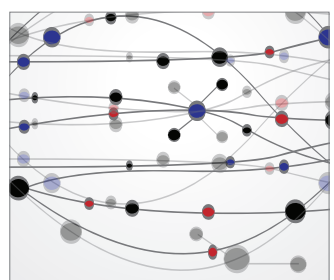

\section{The Scientific} World Journal
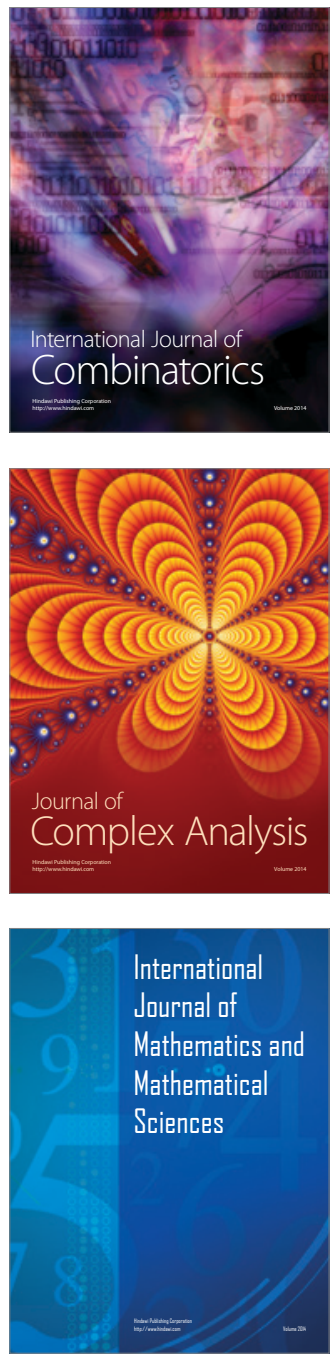
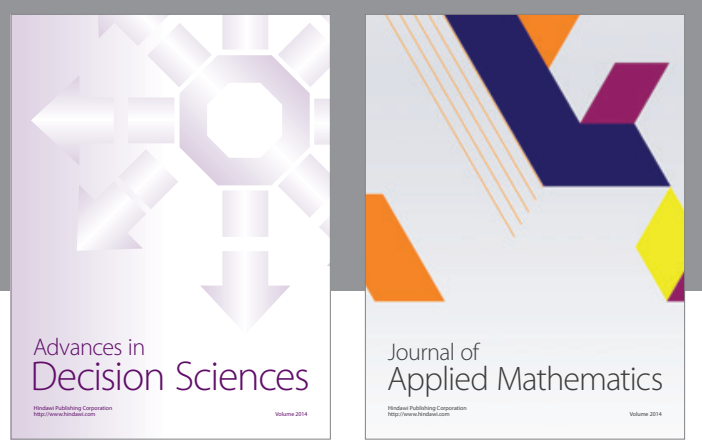

Algebra

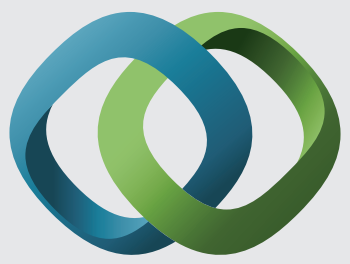

\section{Hindawi}

Submit your manuscripts at

http://www.hindawi.com
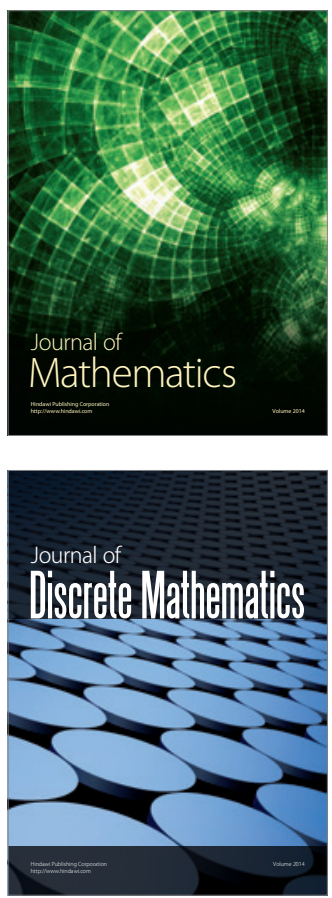

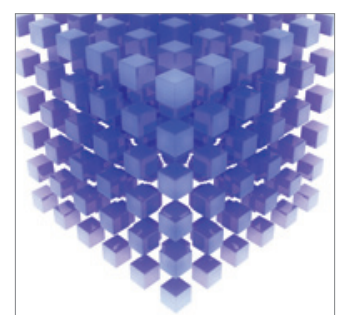

Mathematical Problems in Engineering
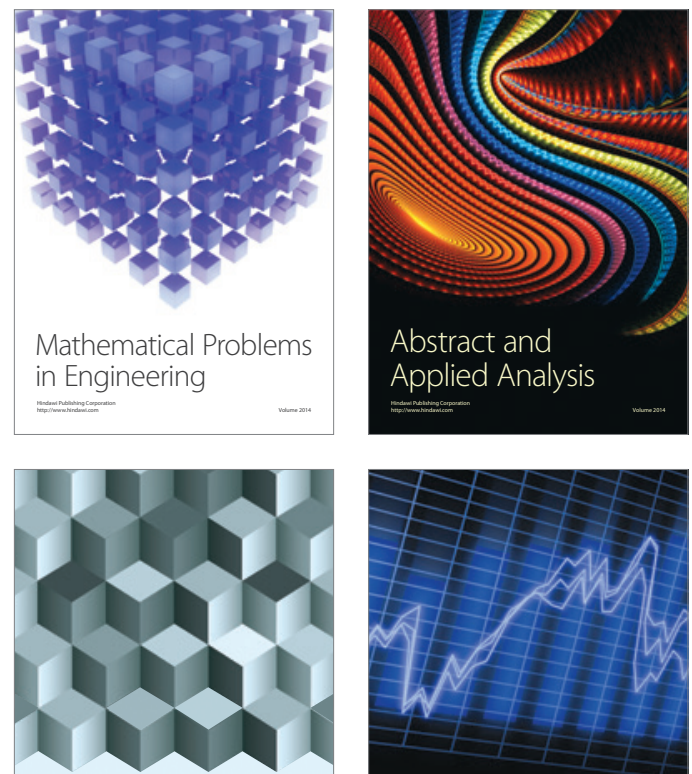

Journal of

Function Spaces

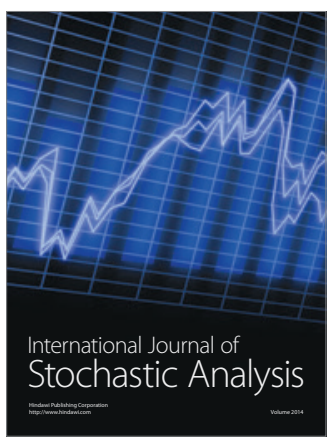

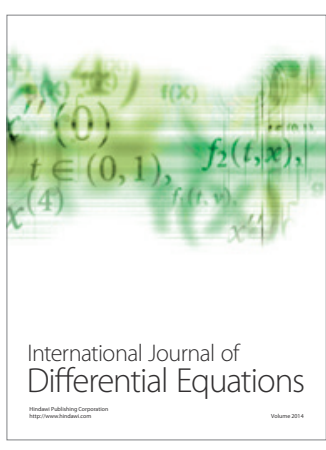
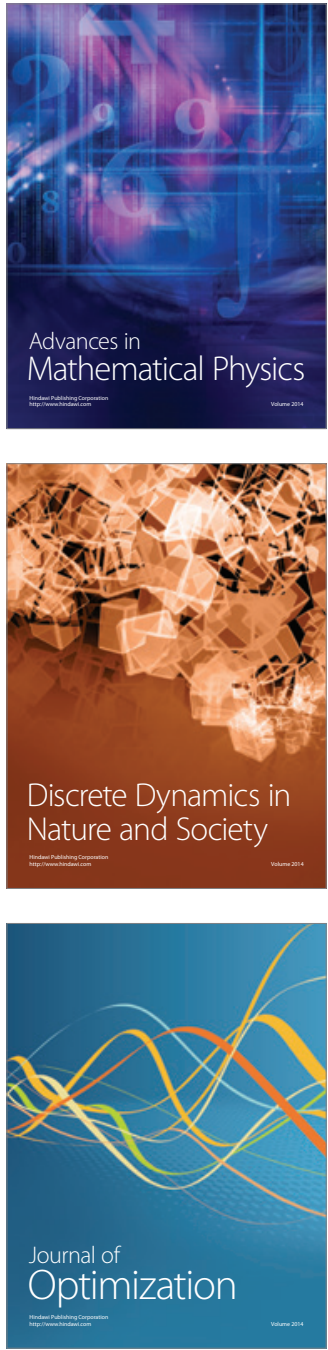